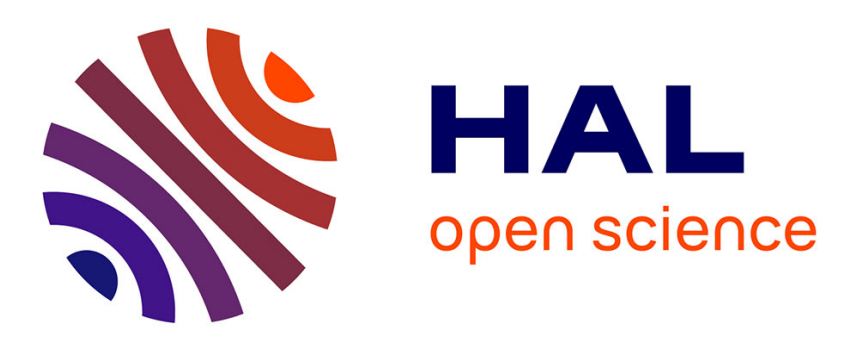

\title{
Contraindre pour aller mieux? Enjeux cliniques et protocolisation dans une unité de prise en charge d'enfants atteints de troubles autistiques
}

\author{
Livia Velpry, Benoît Eyraud
}

\section{- To cite this version:}

Livia Velpry, Benoît Eyraud. Contraindre pour aller mieux? Enjeux cliniques et protocolisation dans une unité de prise en charge d'enfants atteints de troubles autistiques. Demailly Lise, Garnoussi Nadia. Aller mieux. Approches sociologiques, Presses Universitaires du Septentrion, pp.265-278, 2016. halshs-01365542

\author{
HAL Id: halshs-01365542 \\ https://shs.hal.science/halshs-01365542
}

Submitted on 13 Sep 2016

HAL is a multi-disciplinary open access archive for the deposit and dissemination of scientific research documents, whether they are published or not. The documents may come from teaching and research institutions in France or abroad, or from public or private research centers.
L'archive ouverte pluridisciplinaire HAL, est destinée au dépôt et à la diffusion de documents scientifiques de niveau recherche, publiés ou non, émanant des établissements d'enseignement et de recherche français ou étrangers, des laboratoires publics ou privés. 


\title{
Contraindre pour aller mieux? \\ Enjeux cliniques et protocolisation dans une unité de prise en charge d'enfants atteints de troubles autistiques
}

\author{
Livia Velpry, Benoît Eyraud ${ }^{1}$
}

PREPRINT AVANT EPREUVES DE L'ARTICLE PARU :

In Demailly Lise, Nadia Garnoussi (dir.), Aller mieux. Approches sociologiques, Presses Universitaires du Septentrion, Coll. Le regard sociologique, 2016

\section{Correspondance : livia.velpry@univ-paris8.fr}

Les pratiques de soin et les actes médicaux impliquent de nombreuses contraintes sur la personne et le corps du patient. Dans le soin somatique, ces contraintes sont des conditions matérielles qui permettent d'assurer l'acte thérapeutique mais n'ont pas un rôle directement soignant. Le soin en santé mentale se caractérise par le recours à des techniques ayant des dimensions contraignantes auxquelles sont attribuées des effets proprement thérapeutiques. Les techniques de soin très contraignantes abondent dans l'histoire de la psychiatre (Dubreucq, 2012). Certaines cherchent l'effet thérapeutique par le biais organique ; au début du 20ème, les méthodes de choc utilisent ainsi l'intervention parfois violente sur le corps du patient (insulinothérapie, sismothérapie...) pour obtenir une amélioration. L'effet bénéfique relève parfois de la dynamique relationnelle, par exemple lorsque Pinel puis Esquirol préconisent l'autorité du médecin et des contraintes physiques et morales comme outils thérapeutiques du traitement moral. Le caractère thérapeutique de ces techniques a justifié de définir un cadre juridique spécifique qui donne à la contrainte une dimension légale et plus seulement corporelle, ces dimensions étant imbriquées dans l'institution psychiatrique asilaire (Castel, 1977, Postel et Quétel, 2012) .

Spécificité historique, le recours à la contrainte dans le soin en santé mentale a été fortement contesté ces dernières décennies, aussi bien par des acteurs de la société civile que par les psychiatres euxmêmes. Pour autant, le recours à des mesures légales de contrainte augmente depuis une vingtaine d'années ${ }^{2}$ et les dispositifs contraignants (unités très sécurisées, ) sont aujourd'hui fortement financés, justifiés par des impératifs de sécurité ${ }^{3}$. Cette évolution s'accompagne de garanties procédurales et judiciaires ${ }^{4}$ de plus en plus nombreuses, et dont la dimension bureaucratique est indéniable (Demailly, 2014).

Que reste-t-il des revendications thérapeutiques de techniques contraignantes de soin dans un tel contexte? Celles-ci se sont-elles entièrement fondues dans des justifications par les impératifs de sécurité ? Peuvent-elles être encore justifiées pour des raisons cliniques ?

Nous avons déjà cherché à répondre à ces questions dans un contexte d'unités très sécurisées de psychiatrie adulte en soulignant le rôle joué par les notions de "cadre » et de "contenance » pour justifier de la contrainte, et en soulignant les difficultés qu'ont les professionnels à dissocier les motifs de sécurité et les motifs thérapeutiques (Eyraud et Velpry, 2014). Delphine Moreau a souligné le flou entourant les justifications du recours à la contrainte dans le cas de la mise en chambre d'isolement

1 Cet article a été rédigé dans le cadre des travaux menés par le Collectif Contrast (www.contrastcollectif.wordpress.com), financés par l'Agence Nationale pour la Recherche

${ }^{2}$ L'évolution historique du recours à la contrainte n'est pas vraiment objectivable. (Eyraud, Moreau 2013)

${ }^{3}$ Y sont combinés mesure légale de soin sans consentement, portes fermées au sein de l'unité, recours fréquent à la mise en chambre fermée, rigidité des règles de vie collective...

${ }^{4}$ La loi du 5 juillet 2011 modifiée par la loi du 27 septembre 2013, qui autorise les soins sans consentement en psychiatrie, par exception au droit commun du soin, instaure ainsi leur contrôle judiciaire systématique. En outre, des textes de force juridique moindre et aux portées institutionnelles variables (recommandations de bonne pratique, procédures qualité...) encadrent certaines pratiques de soin contraignantes, telles que la mise en chambre d'isolement ou la contention (Collectif Contrast, 2015). 
$(2010)^{5}$. Nous souhaitons dans cette contribution prolonger ces réflexions en nous centrant davantage sur le rôle joué par les règles formelles dans la justification thérapeutique de la contrainte. Nous avons choisi pour cela d'observer un type de soin moins traversé par les controverses autour des usages "sécuritaires" de la contrainte, mais faisant également l'objet de controverses cliniques et scientifiques : la prise en charge en pédopsychiatrie d'enfants atteints de troubles du spectre autistique ayant des troubles du comportement sévères ${ }^{6}$. La prise en charge de l'autisme fait aujourd'hui l'objet d'une attention soutenue des politiques publiques, qui se traduit notamment par des plans d'action publique spécifiés, ainsi que de controverses importantes quant aux modes de prise en charge (Borelle, 2013, Chamak, 2013). L'unité observée s'inscrit dans cette dynamique, sa création relevant des " expérimentations de nouveaux modèles d'accompagnement» promus la mesure 29 du plan autisme ${ }^{7}$. Différentes pratiques réputées contraignantes sont présentes (recours à l'ECT, recours à des chambres spécifiques). Nous accorderons une attention particulière au recours à l'une d'entre elles, la mise en chambre dite « d'hypostimulation ».

Nous montrerons que les professionnels parviennent à revendiquer un usage clinique et thérapeutique de la contrainte, "pour aller mieux», distincts d'un usage à visée de sécurité comme d'un usage maltraitant, en dissociant d'une part fortement pratiques de sécurisation et techniques contraignantes, et en s'appuyant d'autre part sur les règles procédurales qui autorisent le recours aux techniques contraignantes.

Pour cela, nous commencerons par présenter les spécificités de l'unité de soins étudiée et analyserons comment l'impératif de sécurité est dissocié de techniques contraignantes. Puis, nous montrerons comment des techniques contraignantes peuvent être directement investies d'une dimension thérapeutique, en nous intéressant tout particulièrement au rôle de la protocolisation des décisions de mise en chambre « d'hypostimulation ».

\section{La contrainte, à l'articulation de la sécurité et du soin}

Des comportements jugés dangereux sont l'une des difficultés auxquelles les proches ou professionnels s'occupant d'enfants ou adolescents autistes sont confrontés, et qui posent la question du recours à des formes physiques de contrainte. Si pendant longtemps, la psychiatrie était légitime à intervenir sur ces difficultés, cette légitimité a été contestée, conduisant à un travail important de justification du soin psychiatrique autour de l'impératif de sécurité. On va voir dans cette première partie que les outils de sécurisation jouent un rôle central et paradoxal, visant d'une part à dissocier clairement recours à la contrainte et soin, tout en introduisant la possibilité d'un usage thérapeutique de la contrainte.

\section{Une unité expérimentale de prise en charge "thérapeutique" de crise}

L'unité d'hospitalisation étudiée fait partie d'un service de pédopsychiatrie qui accueille des patients mineurs en hospitalisation libre autorisée par leurs parents ou tuteur. Spécialisée dans l'accueil des situations de crise et d'urgence, sa création s'est faite par réponse à un appel à projet de l'ARS, réponse centrée sur un projet expérimental de «prise en charge multidisciplinaire, intégrative des personnes

\footnotetext{
5 Ceux-ci maintiennent un flou sur les motifs de l'usage des techniques contraignantes, dont il est parfois difficile de savoir s'il s'agit d'une punition, d'un soin ou d'une gestion des contraintes organisationnelles du travail. Conserver l'ambiguïté évite d'expliciter la spécificité des motifs cliniques qui justifient de la mise en chambre fermée et qui reposent sur le paradoxe d'un recours à la contrainte avec la finalité que la personne aille mieux.

${ }^{6}$ Nous nous appuyons sur des observations réalisées dans l'unité au cours de 6 mois de présence intermittente et sur des entretiens réalisés avec les professionnels de l'unité.

${ }^{7}$ Ce faisant, le travail réalisé au sein de l'unité se trouve exposé à la critique, aussi bien en raison des controverses autour du rôle de la psychiatrie dans la prise en charge de l'autisme, que par la place des parents ou tuteurs, qui doivent donner leur consentement aux soins. Alors que les arbitrages et processus de justification auxquels donne lieu le recours à la contrainte dans les pratiques de soin en santé mentale sont souvent masqués et résiduels. Dans cette unité, du fait notamment de ce contexte à la fois souple et très surveillé, ils sont rendus particulièrement visibles.
} 
autistes en situation de décompensation ». Cette dimension expérimentale implique que l'unité soit privilégiée par rapport à d'autres structures en termes d'encadrement, celle-ci recevant un nombre limité de patients - entre sept et neuf au moment de l'observation- pour une dotation en personnelle importante, et par des ressources qui permettent la réfection des locaux endommagés par les patients comme l'achat de matériel de protection. Le public cible est ordinairement déjà pris en charge dans des institutions médico-sociales ou par les parents au domicile, l'hospitalisation étant justifiée quand ces institutions sont dépassées par des comportements hétéro-agressifs, auto-agressifs ou perturbants particulièrement importants ${ }^{8}$. L'hospitalisation dans cette unité expérimentale a vocation à réduire ces troubles du comportement afin de permettre le retour à une situation de prise en charge stable, ce qui passe par trouver une réponse clinique pertinente aux troubles du comportement. L'équipe met en œuvre une double approche thérapeutique ${ }^{9}$. Dans sa dimension médicale, la prise en charge consiste à ajuster les traitements médicamenteux et à traiter les affections somatiques. Ces dernières sont parfois à l'origine des troubles du comportement, en causant des douleurs que le patient ne réussit pas à exprimer mais qui le conduisent à être hétéro ou autoagressif. En effet, pour presque tous les patients accueillis, le trouble autistique s'accompagne d'un retard mental important et de troubles de la communication, avec un accès limité au langage oral. Dans sa dimension psycho-éducative, l'intervention vise la diminution ou la disparition des troubles du comportement, envisagés comme une réponse inadaptée dans une situation donnée. Cette approche suppose d'en identifier les mécanismes d'apparition, souvent associés aux difficultés de communication ${ }^{10}$.

\section{Sécuriser pour réduire la contrainte : des techniques peu contraignantes de sécurisation}

Au cours des six mois d'observation, plusieurs patients sont admis à l'unité après avoir été hospitalisés dans un service de psychiatrie adulte, où ils ont passé plusieurs semaines, voire plusieurs mois en isolement, à des fins de protection du patient comme de l'entourage. D'autres ont été placés sous contention physique en institution ou à domicile, dans le but de limiter les comportements hétéro et auto-agressifs et leurs conséquences. Les situations de vie ou de prise en charge que quittent les patients lorsqu'ils sont admis à l'unité sont donc souvent très contraignantes et perçue comme maltraitantes y compris par les professionnels ou parents qui les mettent en œuvre.

Lors de l'admission, les professionnels de l'unité cherchent d'abord des réponses plus acceptables aux troubles de comportements des patients, tant en terme de degré de contrainte que de pertinence clinique. Pour cela, la première étape consiste à dissocier l'objectif de sécurisation de l'objectif thérapeutique dans la réponse apportée au trouble de comportement. La sécurité des professionnels et celle des patients sont ainsi assurées par divers moyens de protection physiques, ce qui permet de diminuer les interventions contraignantes en direction du patient à des fins de sécurité. Ainsi, face aux griffures profondes et soudaines qu'inflige une jeune patiente hospitalisée, le médecin introduit le port de mitaines pour les professionnels. Cet outil sécurise en protégeant des blessures et évite dans le même temps de recourir à la contention des mains de la patiente. De même, l'usage du casque,, que les patients peuvent enlever seuls, permet aux patients de ne pas se faire trop mal quand ils se tapent la tête contre les murs, d'éviter que les coups violents ne les mettent en danger ou aient des conséquences trop graves. Ainsi, les soignants distinguent fortement les outils de sécurisations utilisées envers ces

\footnotetext{
${ }^{8}$ Un document de présentation de l'unité liste les troubles du comportement les plus fréquents : «automutilation, agressivité, agitation, destruction, pica, fugue/chutes». Le pica désigne un trouble qui consiste à ingérer des éléments non comestibles.

${ }^{9}$ L'équipe soignante comprend des médecins, infirmiers et aides-soignants du côté médical, et des psychologues, éducateurs, orthophoniste, psychométricienne, moniteurs-éducateurs du côté psycho-éducatif. La prise en charge définie pour un patient est cependant mise en œuvre par l'ensemble de l'équipe.

${ }^{10}$ L'évaluation précise des capacités de communication, expression comme compréhension, et l'utilisation d'outils de communication adaptés constitue une part importante de la prise en charge. Ainsi, face à un patient qui renverse une table contre le mur pendant une activité, les soignants peuvent interpréter ce geste comme la seule façon qu'a le patient de signifier qu'il souhaite arrêter l'activité. L'objectif thérapeutique consistera alors à lui apprendre à exprimer un «non » et à observer ensuite si le comportement disparaît.
} 
patients avant leur entrée à l'unité, qui peuvent être contraignantes et maltraitantes, des outils de sécurisation spécialisés qui permettent de réduire le recours à des techniques contraignantes.

\section{L'introduction de la contrainte comme thérapeutique : l'exemple du casque}

Pour autant, ces techniques de sécurisation peuvent être ambiguës : d'une part, parce qu'elles peuvent être plus ou moins contraignantes ; d'autre part, parce qu'elles peuvent être justifiées également pour des raisons thérapeutiques, comme on va le voir avec l'exemple du casque de Zoé.

Zoé a 13 ans. Au moment où je la rencontre, elle est hospitalisée depuis plusieurs semaines. La plus grande partie de la journée, elle porte un casque rigide, qui ressemble à un casque de surf. Dans les moments où ses comportements deviennent trop intenses et si elle ôte son casque, les soignants lui mettent alors un autre casque, avec une grille qui protège le visage, que Zoé ne peut pas enlever seule. C'est ce qui se produit un jour où, alors qu'elle est placée en chambre fermée, Zoé enlève son casque rigide et se tape violemment la tête contre le mur. Deux soignants interviennent immédiatement en apportant le casque à grille qu'ils tentent de lui mettre malgré ses résistances. Le recours à la force est justifié par le risque qu'a Zoé de se faire mal. Pour autant, il est compatible avec la recherche de coopération. Ainsi, alors que Zoé s'agite, les soignants maintiennent ses mains et ses pieds pour tenter de l'immobiliser afin de lui mettre le casque. Dans le même temps, l'infirmier qui lui tient les mains lui parle, l'incitant à se calmer et lui promettant de lui lire une histoire ensuite. Malgré cette double stratégie, Zoé continue à s'agiter. La situation se résout quand, quelques minutes plus tard, l'autre soignant demande à Zoé de les aider à passer son casque. Cette dernière cesse alors de se débattre, l'infirmier lui lâche bras et jambes, et Zoé met elle-même le casque, que les soignants attachent.

Les différents casques ont tous une fonction de sécurisation vis à vis du comportement auto-agressif de Zoé, mais leur usage est différencié et s'articule au fait que Zoé « va mieux » ou pas. Au moment où cette scène se produit, Zoé porte en permanence son casque rigide, ce qui signifie, pour l'équipe, que Zoé «va mieux » : à la différence de ce qui se produisait il y a quelques mois à son arrivée, elle ne se tape plus que rarement la tête contre les murs. Le casque à grille ne lui est plus proposé, ou mis de force comme dans le cas évoqué plus haut, que dans des moments de crise. Si Zoé continue à " aller mieux», l'étape suivante consistera à échanger ce casque rigide contre un casque souple, pour envisager ensuite éventuellement de s'en passer totalement. Mais cette transition doit être acceptée par Zoé. Le passage au casque souple diminue la sécurisation vis à vis de ses comportements, et on ne peut donc le lui imposer, au risque de déclencher une augmentation de ces comportements d'auto-agression. En effet, Zoé refuse les propositions des soignants d'enlever le casque rigide ; elle semble se sentir protégée par ce casque.

L'objectif clinique des soignants est ainsi qu'elle supporte cette diminution de la contrainte. Pour cela, la consigne générale est de tenter de faire accepter à Zoé des petits temps sans son casque rigide, afin de la déshabituer peu à peu. Des stratégies visent de contourner la demande de Zoé de garder son casque. Par exemple, lors du repas, pendant lequel Zoé a l'habitude d'enlever son casque, un des soignants le soustraie à sa vue et attend qu'elle le réclame plutôt que de lui proposer, espérant allonger ainsi le temps qu'elle passe sans casque. Casque à grille, casque rigide ou casque souple, les passages de l'un à l'autre de ces outils de sécurisation constituent ainsi à la fois des indicateurs de l'amélioration de l'état de Zoé et des objets du travail thérapeutique réalisé par les soignants.

Ceux-ci justifient le recours à la contrainte comme un moyen pour atteindre l'horizon idéal de la relation thérapeutique consentie. Il a donc vocation à être dépassé en permettant l'acquisition de l'autonomie (Velpry, 2008), et la reconnaissance a posteriori, par la personne, de la nécessité de la contrainte dont elle a fait l'objet.

Ainsi, les outils de sécurisation des personnes jouent un rôle central : d'une part, ils permettent de justifier d'une réduction de la contrainte au regard de ce qui était vécu auparavant par les patients ; d'autre part, en laissant les comportements des patients s'exprimer, ils permettent aux professionnels de les observer, de tester des manières d'y répondre, et d'introduire une dimension thérapeutique, qui n'échappe pas à l'ambiguïté d'une forme d'injonction à l'autocontrainte. Cette position ambiguë du 
recours à la contrainte rend d'autant plus important le travail effectué par les professionnels, dans le cours de leurs pratiques, pour le façonner au croisement de l'encadrement légal, des justifications thérapeutiques et des organisations institutionnelles.

Nous allons poursuivre l'analyse en nous intéressant à la place prise par le protocole pour éviter que les situations contraignantes ne soient maltraitantes.

\section{La protocolisation du recours à la contrainte : l'exemple de la chambre d'hypostimulation}

Lorsque les techniques envisagées sont contraignantes, les équipes doivent effectuer un travail permanent pour s'assurer d'en avoir un usage thérapeutique et l'associer à une évolution de l'état de la personne. Nous faisons l'hypothèse dans cette seconde partie que l'usage des règles formalisées, au niveau des principes généraux comme de leur spécification, participe de ce travail de réflexivité garantissant la justification thérapeutique du recours à la contrainte. Toutes les pratiques contraignantes ne font toutefois pas l'objet d'une formalisation. Le port du casque ne fait ainsi pas l'objet de formalisation particulière.

Nous nous intéressons donc à une pratique qui peut faire l'objet de différentes formalisations des règles, à savoir mise en chambre spéciale, nommée dans l'unité observée "chambre d'hypostimulation ». Cette pratique ne fait pas consensus dans le champ des interventions thérapeutiques envers les patients autistes ${ }^{11}$. Sa mise en œuvre dans une unité nouvelle s'appuie sur un certain nombre de règles juridiques et de recommandations liées à la littérature médicale, et qui ont été depuis lors intégrées au cahier des charges. Après avoir présentées les règles " extérieures » qui sont suivies à l'intérieur du service, nous montrerons comment celles-ci sont mobilisées afin de garantir une dimension « thérapeutique » et non « maltraitante » au recours à la chambre d'hypostimulation.

Règles juridiques, préconisations scientifiques, et recommandations relatives à la chambre d'hypostimulation

$\mathrm{Du}$ point de vue juridique, la mise en chambre d'hypostimulation fait l'objet d'une prescription médicale, qui figure dans le dossier du patient. Pour les mineurs hospitalisés librement par leurs parents, ceux-ci sont informés par le médecin.

Les modalités précises d'usage de cette pratique se rattachent à au moins deux justifications d'un point de vue clinique. La première renvoie aux «troubles de la modulation sensorielle » qui caractérisent fréquemment les troubles autistiques dans le consensus actuel (HAS, 2010) ${ }^{12}$ et auquel fait directement référence le terme "hypostimulation ». Dans les publications scientifiques, des auteurs associent la difficulté à gérer les stimulations sensorielles à la survenue de «comportements-problèmes » (Degenne-Richard, 2014). Dans cette perspective, la mise en chambre d'hypostimulation permet à la personne de s'apaiser en la plaçant dans un environnement de moindre stimulation sensorielle ${ }^{13}$.

En second lieu, la pratique qui consiste à isoler un patient à la suite d'un comportement problématique s'inscrit pleinement dans le répertoire des méthodes de traitement comportementales de l'autisme, à condition que le temps de mise à l'écart soit court. La méthode $A B A$, qui en représente une version très formalisée, mise en avant par certaines associations de parents à l'appui de leur contestation des approches d'inspiration psychanalytique, la désigne sous le terme de « time out». Le principe consiste à répondre à un comportement inapproprié en supprimant l'accès à des "renforçateurs positifs », afin que la personne renonce peu à peu à ce comportement (Cooper, Heron et Heward, 2007). La mise en

\footnotetext{
${ }^{11}$ Rapport HAS, p.232

12 Ils font partie des critères diagnostiques des troubles du spectre autistique dans la dernière version du manuel diagnostique américain, le DSM5.

13 Il n'existe pas de recommandation de bonne pratique concernant la mise en chambre fermée de mineurs en hospitalisation libre en France. Un rapport suisse (Bovet P., 2009) mentionne uniquement l'existence de recommandations de l'American Academy of Child and Adolescent Psychiatry, aux Etats-Unis, datant de 2002.
} 
chambre fermée vise à soustraire la personne à ses « renforçateurs ». Dans le contexte très controversé du traitement de l'autisme (Chamak, 2011), la mise en chambre d'hypostimulation a ainsi la particularité et l'intérêt de voir son mécanisme d'action thérapeutique et ses modalités d'usage déterminées par deux approches différentes. Dans l'unité, les professionnels se réfèrent d'ailleurs à l'un comme à l'autre lors des discussions.

Pour de nombreux patients, cette justification scientifique, et la prescription médicale sont accompagnées de protocoles individualisés, élaborés de manière collégiale par le médecin et l'équipe de soignants. L'élaboration d'un protocole individualisé est préconisée par les recommandations de la HAS. De fait, l'individualisation vise à répondre aux difficultés rencontrées au moment où se posent la pertinence du recours à cette pratique : doutes exprimés par les soignants au moment d'identifier ce qui justifie, ou pas, une mise en chambre d'hypostimulation; dissension forte entre le médecin et les parents sur l'usage de la pratique; ou encore d'incertitudes sur les effets et bénéfices de la pratique pour le patient.

Dans les pratiques, la manière de suivre règles juridiques et recommandations scientifiques varie. Ces situations montrent que les deux usages du protocole, pour justifier un écart aux règles générales et pour assurer une application homogène, s'inscrivent tous deux dans la préoccupation de préserver le caractère thérapeutique de la pratique.

\section{Une prescription standardisée : expérience de l'aller mieux et routinisation du recours à la contrainte}

Zoé est assise dans le couloir avec son casque rigide. Elle se tape la tête contre le mur. Guy lui dit, tu le fais encore une fois et tu vas en chambre d'hypostimulation. Tu veux? Zoé répond non. Elle recommence à se taper la tête contre les murs. Guy lui demande, tu veux rester ici ou aller en chambre d'hypostimulation? Zoé se débat, Guy la prend par le bras sans hésitation et l'emmène en chambre d'hypostimulation. Quelques minutes plus tard, il vient ouvrir la porte et Zoé ressort plus calme. (Journal de terrain)

Cette scène se fond dans la routine de l'unité. Il arrive régulièrement à Zoé de faire ce geste, ou alors de jeter quelque chose ou encore de se diriger vers quelqu'un pour le taper. Souvent, on n'identifie aucun élément déclencheur à son action. En réponse à ces comportements, elle est mise immédiatement dans la chambre d'hypostimulation, par un des membres de l'équipe qui se trouve présent, ici Guy, un moniteur-éducateur, celui-ci établissant implicitement un diagnostic (autoagressivité violente, comportement inapproprié ) qui justifie la mise à l'écart contrainte.

Une fois placée dans cette pièce fermée à clef, elle y est surveillée régulièrement par une vitre. Elle y reste un temps court - de 5 à 15 minutes environ -, au terme duquel elle sort généralement plus calme. Dans la situation décrite, Zoé porte son casque rigide et ne risque pas de se faire vraiment mal. Sa mise en chambre vise à l'apaiser, et est conforme à la prescription médicale générale, qui a été faite à l'entrée de la patient dans le service, que les soignants ne consultent même pas dans la plupart des mises en chambre de Zoé. La répétition des mises en chambre avec effet d'apaisement a suffi à justifier la routinisation de cette pratique. Conforme aux règles juridique et clinique qui autorisent cette pratique au sein du service, l'usage qui est fait de la chambre d'hypostimulation pour Zoé est de ce fait rarement explicité dans le quotidien de l'équipe et n'a pas fait l'objet d'un protocole individualisé.

Cette absence de discussion et d'individualisation ne correspond pas aux recommandations de la HAS. Pour autant, elle n'est pas exempte d'une dimension thérapeutique, le constat partagé de manière informelle que Zoé va mieux en sortant de la chambre permettant justement d'inscrire cette pratique dans le projet de l'unité et de la routiniser comme soignante. L'expérience clinique de l'aller-mieux permet alors cette routinisation, mais celle-ci n'est pas exempte de risque d'ambiguïté14.

\footnotetext{
${ }^{14}$ De ce fait, dans le cas de Zoé, les motifs de mise en chambre d'hypostimulation peuvent comporter une certaine ambiguité - telle une mise en chambre après que Zoé jette brusquement son assiette de carotte râpées sur un soignant, qui pourrait être influencée par l'envie de "marquer le coup » ou l'exaspération après les trois coups violents que Zoé a donnés dans la matinée, ou encore viser aussi à "soulager la pression" dans la salle à manger où plusieurs autres patients viennent de s'agiter -, sans que cela ne soulève véritablement de doute sur le caractère éthique et thérapeutique de la décision.
} 


\section{Une individualisation dérogatoire du protocole : l'exemple de Roman}

Le protocole de mise en chambre d'hypostimulation réalisé pour Roman précise qu'il faut l'y placer en prévention des crises, plusieurs fois par jour, pour des temps longs. Cet usage est initié face aux problèmes que posent les troubles de comportement de Roman. De très grande intensité, les accès de violence de Roman mettent en difficulté les professionnels de l'unité, malgré les protections physiques et leur savoir-faire pour maîtriser les agitations, provoquant de nombreux arrêts-maladie. Non seulement les mises en chambre d'hypostimulation lors des crises sont difficiles et dangereuses en raison de sa force physique, mais leur effet est douteux. Elles semblent accroître son agitation plutôt que la diminuer, décuplant sa force, ce qui l'amène plusieurs fois à briser la porte. La mise en chambre d'hypostimulation ne permet pas de sécuriser Roman et les autres personnes présentes, ni d'améliorer son état.

Mis en chambre d'hypostimulation de façon préventive et pour des temps longs, Roman a moins fréquemment des accès de violence. Professionnels et patients sont ainsi sécurisés, mais le caractère éthique et thérapeutique de cet usage particulier reste fragile. En effet, le nouvel usage institué est exactement contraire aux préconisations cliniques, qui précisent qu'il doit être en réaction à un comportement et pour des temps courts. C'est donc une explication clinique spécifique, portée par le médecin-chef de l'unité, qui justifie le protocole de Roman. Elle combine une analyse des mécanismes des troubles du comportement de Roman avec l'hypothèse de la modulation sensorielle. Dans cette explication, Roman gère particulièrement mal les stimulations sensorielles, accumule les tensions qui s'expriment ensuite avec violence. Le séjour préventif prolongé en chambre d'hypostimulation maintient un niveau de stimulation plus acceptable. Outre cette justification clinique, l'observation des effets du protocole sur l'état de Roman et les tentatives de diminuer le temps passé en chambre d'hypostimulation participent à l'inscrire dans une intervention thérapeutique.

\section{L'importance de suivre le protocole à la lettre : l'exemple de Baptiste}

Le protocole rédigé pour Baptiste précise que la mise en chambre d'hypostimulation doit intervenir de façon systématique, pour tout trouble du comportement, et qu'elle doit durer exactement cinq minutes. Il mentionne également qu'un minuteur doit être accroché la vitre, afin que Baptiste puisse y suivre l'écoulement du temps prescrit. Un tel protocole ne déroge pas aux règles habituelles d'usage de la chambre d'hypostimulation. Quelle est alors la nécessité de le rédiger et de l'afficher dans le poste de soin, au vu de tous?

Dans ses premières semaines dans l'unité, Baptiste frappe très souvent professionnels et patients, sans déclencheur apparent. De façon plus inhabituelle par rapport aux autres patients, il " fait des bêtises ", ce par quoi les soignants désignent des comportements perturbants incessants qu'ils perçoivent comme de la provocation. Baptiste est très fréquemment mis en chambre d'hypostimulation, alors même que l'incertitude grandit sur la pertinence de cette pratique. Tout d'abord, les «bêtises » de Baptiste relèvent-elles d'une provocation ou d'un trouble du comportement, et comment les distinguer afin d'avoir des réponses différenciées ? Les éducatrices insistent sur l'importance d'une réponse unifiée des soignants si Baptiste cherche effectivement à provoquer. Mais ce que les soignants perçoivent comme des «bêtises" l'est-il vraiment? L'orthophoniste alerte sur ses capacités de communication, qui seraient beaucoup plus faibles en réalité qu'elles ne le paraissent. Dans ce cas Baptiste ne serait pas dans la provocation, malgré les apparences ; il n'en serait pas capable. Il exprimerait quelque chose par ses troubles du comportement, par exemple des douleurs somatiques. Non seulement la mise en chambre d'hypostimulation ne serait pas soignante, mais elle pourrait même être maltraitante en instaurant une punition qu'il ne peut pas comprendre. En outre, les effets sont-ils bénéfiques? Les moments passés en chambre ne semblent pas apaiser Baptiste mais au contraire générer des troubles auto-agressifs, cependant qu'au fil de jours, la fréquence de survenue de ses troubles semble augmenter.

Face aux doutes sur la nature des comportements perturbateurs et sur les capacités de compréhension de Baptiste, le protocole élaboré en réunion d'équipe vise à la fois à tester une explication clinique et à 
écarter le risque de maltraitance. Il instaure une réaction systématique aux troubles du comportement ${ }^{15}$ et prévoit une évaluation de son effet pendant la semaine par les professionnels. Si les effets sur les troubles du comportement de Baptiste sont perçus comme positifs, la pertinence d'une sanction systématique dans le cadre d'une réponse éducative sera renforcée. En fixant un temps précis et court avec minuteur, le protocole veut s'assurer que Baptiste conserve un peu de maîtrise sur la situation en en comprenant la durée. Ainsi, le protocole de Baptiste réaffirme le caractère thérapeutique de la mise en chambre d'hypostimulation mais plutôt en raison d'un usage effectif des soignants dévié par rapport à son objectif initial.

Dans ce cas, la prescription médicale standard ne suffit plus à légitimer l'usage ; il faut redéfinir les critères de mise en chambre à partir des objectifs thérapeutiques, expliciter ses modalités précises, et en observer les effets. Pour s'assurer du caractère thérapeutique de l'usage, un processus de transformation de la règle est donc nécessaire.

Le recours à la chambre d'hypostimulation est une pratique contraignante de soin dont la dimension thérapeutique ne fait pas consensus. Pour justifier d'une telle pratique, les équipes médicales s'appuient sur une littérature scientifique qui en définit les critères d'application, ainsi que sur des recommandations de bonne pratique, qui énoncent l'importance d'individualiser ces protocoles pour garantir une dimension thérapeutique au soin. L'observation de ces pratiques nous a permis de distinguer trois types d'usages des règles formalisées montrant que la justification thérapeutique de la contrainte imbrique des dimensions d'expérience permettant une forme de dispense d'individualisation du protocole, de respect à la lettre de la règle, ou au contraire d'usage dérogatoire dont la justification doit être explicitée.

En pointant la distance qui existe parfois entre les recommandations de bonne pratique et les pratiques effectives, par exemple concernant le fait de ne pas systématiquement recourir à un protocole individualisé additionnel, nous ne cherchons pas à émettre un jugement sur sa pertinence. L'analyse sociologique éclaire l'interdépendance entre les situations où les règles plus générales, cliniques et juridiques, suffisent à justifier le recours à la contrainte, et celles où une spécification est nécessaire. L'individualisation du protocole ne suffit pas à garantir l'effet thérapeutique.

\section{Conclusion}

La hausse des mesures légales autorisant le soin sous contrainte en psychiatrie et le développement de dispositifs très sécurisés font l'objet de vives critiques qui reçoivent notamment comme réponse l'augmentation des garanties procédurales apportées à ces soins.

Nous nous sommes demandés à travers cet article si, dans un tel contexte, la justification thérapeutique, "pour aller mieux», de pratiques contraignantes parvenait à trouver place. Nous avons cherché à éclairer comment une telle justification peut s'adosser à une protocolisation des pratiques de soin.

En choisissant une unité expérimentale de prise en charge multidisciplinaire et intégrative des personnes autistes en situation de décompensation, nous avons montré dans une première partie que la justification thérapeutique de la contrainte est imbriquée avec les outils spécialisés de sécurisation. Ceux-ci permettent de réduire la contrainte reçue avant leur hospitalisation par des patients aux comportements violents; inversement, leur appropriation thérapeutique peut justifier qu'ils soient imposés par les soignants, ce qui ouvre alors la porte à une incertitude sur la légitimité du recours à ces outils de sécurisation.

Pour répondre à cette incertitude, des procédures sont élaborées concernant l'usage des outils de sécurisation les plus contraignants. Nous avons choisi dans une seconde partie d'analyser cette procéduralisation en observant le suivi des règles dans des décisions de mise en chambre d'hypostimulation. Nous avons montré que cette pratique fait l'objet de règles juridiques, de règles de prescriptions médicales, et de recommandations portant sur l'individualisation de protocoles. Les différents exemples étudiés ont souligné que le souci thérapeutique de l'aller-mieux se loge dans la

${ }^{15}$ L'affichage dans l'unité en plusieurs exemplaires et son rappel oral fréquent participent de cette recherche de réponse systématique et constituent donc un outil clinique. 
tension entre la règle générale de recours à la contrainte et sa spécification. Nous avons montré que cette tension peut se dénouer par expérience, et conduire à une routinisation du recours à la contrainte ; ou par une individualisation de la règle générale se traduisant par la possibilité d'élaborer des dérogations à la règle, ou au contraire d'en exiger un suivi strict. Plus qu'une procédure de contrôle bureaucratique, la formalisation de règles entourant les pratiques contraignantes semble encourager, dans cette unité expérimentale, une réflexivité thérapeutique.

\section{Références}

Borelle C. (2013), Le traitement social de l'autisme. Étude sociologique du diagnostic médical. Thèse pour l'obtention du grade de docteur en sociologie, IEP de Grenoble

Bové, P. (2009). Mesures de contention dans les hôpitaux psychiatriques du Canton de Vaud, Rapport adressé à M. le Secrétaire général du DSAS, Service de psychiatrie générale, Centre Hospitalier du Nord Vaudois, Lausanne

Castel, R. (1977). L'ordre psychiatrique. L'âge d'or de l'aliénisme. Paris: Ed. de Minuit.

Chamak, B. (2013). Autisme: nouvelles représentations et controverses, Psychologie clinique, 36, 59-67

Collectif Contrast (2015) La régulation des pratiques contraignantes de soin en santé mentale: perspectives pour une approche interdisciplinaire, dans Koubi G., Hennion-Jacquet P., Azimi V., L'institution psychiatrique au prisme du droit. Ed. Panthéon-Assas

Cooper, J., Heron, T., \& Heward, W. (2007). Applied Behaviour Analysis. New Jersey: Pearson Education.

Degenne-Richard, C. (2014), Evaluation de la symptomatologie sensorielle des personnes adultes avec autisme et incidence des particularités sensorielles sur l'émergence des troubles du comportement, Thèse pour l'obtention du doctorat de Psychologie, Université Paris Descartes

Demailly, L. (2014). Variations de la 'démocratie sanitaire' et politique publique de santé mentale en France, SociologieS [En ligne], Théories et recherches, URL : http://sociologies.revues.org/4653

Dubreucq, J. (2012). La contrainte, un outil de soin en psychiatrie?, Thèse pour l'obtention du doctorat en médecine, Univ. Joseph Fourier, Faculté de médecine de Grenoble

Eyraud, B. et Moreau D. (2013). Formes et régulations de l'enfermement psychiatrique : de la création de l'asile aux nouvelles unités sécurisées, l'exemple de l'hôpital du Vinatier. Cultures et conflits, 90

Eyraud, B. et Velpry L. (2014). Redéfinir la place de l'enfermement dans la pratique psychiatrique, l'exemple des unités sécurisées, in Bujon Thomas, Dourlens Christine, Le Naour Gwenola, Aux frontières de la médecine, Editions des Archives contemporaines

HAS, (2010). Autisme et autres troubles envahissants du développement. Etat des connaissances hors mécanismes physiopathologiques, psychopathologiques et recherche fondamentale, Argumentaire

Henckes, N. (2008). Réformer et soigner, L'émergence de la psychothérapie institutionnelle en France, 1944-1955, dans J. Arveiller (dir.), Psychiatries dans l'histoire, Caen, PUC, 277-288

Moreau D. (2013). Contraindre pour soigner ? Le care à l'épreuve de la contrainte dans un service d'hospitalisation psychiatrique, dans C. Crignon et M. Gaille (dir.), Qu'est-ce qu'un bon patient ? Qu'estce qu'un bon médecin ?, Paris, éd. Seli Arslan

Postel J., Quétel C., Nouvelle histoire de la folie, Dunod, 2012

Velpry, L. (2008). Le quotidien de la psychiatre, Paris, Armand Colin

Velpry, L., Eyraud B. et al. (2014). Réguler les pratiques contraignantes de soin en santé mentale: recompositions et enjeux, dans Jean-Charles Pascal et Cécile Hanon (dir.), Consentement et contrainte dans les soins en psychiatrie, Paris, Doin Editions 